\title{
Approach for Transforming Monolingual Text Corpus into XML Corpus
}

\author{
Deepak Sharma \\ Student \\ Department of Information Technology \\ Bharati Vidyapeeth College of Engineering \& Research, \\ Pune 411043, India
}

\author{
Prakash.R.Devale \\ Associate Professor \\ Department of Information Technology \\ Bharati Vidyapeeth College of Engineering \& Research, \\ Pune 411043, India
}

\begin{abstract}
In this paper, we are presenting the approach to convert the text based monolingual corpus to Part-Of-Speech tagging using an standard tagging tool in tagged file and then convert tagged file in the XML format as per defined DTD (Document Type Definition). The tagged text document is parsed through the logic to generate the corpus in XML and also, it can be further used for Information Retrieval, Text-To-Speech conversion, Word Sense Disambiguation and also useful for preprocessing step of parsing by providing unique tag to each word which reduces the number of parses.
\end{abstract}

\section{General Terms}

Natural Language Processing

\section{Keywords}

Part-Of-Speech tagging, Java XML library, DOM Parser.

\section{INTRODUCTION}

In corpus linguistics, part-of-speech tagging (POS tagging), also called word-category disambiguation or grammatical tagging, is the process of marking up a word in a text (corpus) as corresponding to a particular part of speech, based on both its definition, as well as its context-i.e. relationship with adjacent and related words in a phrase, sentence, or paragraph. A simplified form of this is commonly taught to school-age children, in the identification of words as nouns, verbs, adjectives, adverbs, etc.

Once performed by hand, POS tagging is now done in the context of computational linguistics, using algorithms which associate discrete terms, as well as hidden parts of speech, in accordance with a set of descriptive tags.

Definition: The process of assigning a part-of-speech or other lexical class marker or tags to each word in a corpus is called Part-Of-Speech. Figure 1.1 shows the example of Part-OfSpeech tagging.

In this paper, we have implementing the logic to build the corpus in XML as per user defined DTD for any

\section{CLASSIFICATION OF PART-OF- SPEECH TAGGING}

The approach for Part-Of-Speech tagging is classified into three types as follows:[1]

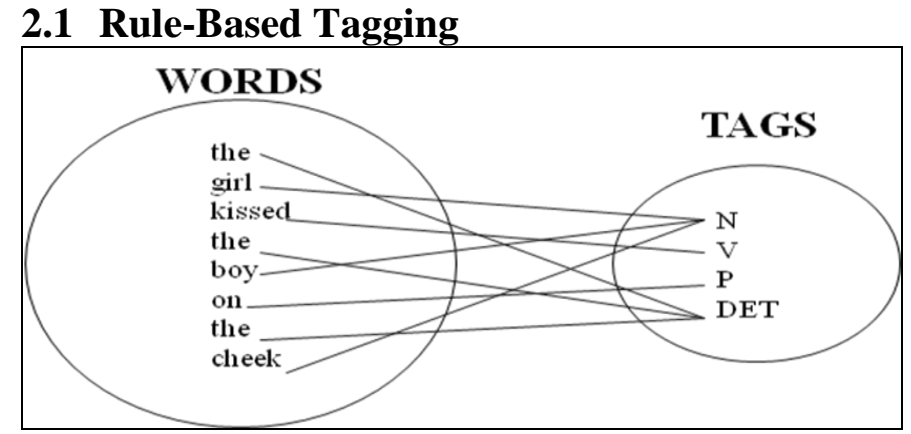

Figure 1.1: Example of Part-Of-Speech Tags.

The basic idea behind in Rule based Tagging is to assign all possible tags to all words. Remove tags according to set of rules of type: if word +1 is an adj, adv, or quantifier and the following is a sentence boundary and word- 1 is not a verb like "consider" then eliminate non-adv else eliminate adv. Typically more than 1000 hand-written rules, but may be machine-learned.

\subsection{Stochastic Tagging}

Based on probability of certain tag occurring given various possibilities. In this approach for pos-tagging, it requires a training corpus. There would be no probabilities for words not in corpus. Also, Training corpus may be different from test corpus. For stochastic tagging, choose most frequent tag in training text for each word.

\subsection{Transformation-Based Tagging}

It is also as Brill-Tagging. This approach is a combination of Rules-based and Stochastic tagging methodologies as:

a) Like rule-based because rules are used to specify tags in a certain environment.

b) Like stochastic approach because machine learning is used - with tagged corpus as input

The basic idea behind this approach as it Set the most probable tag for each word as a start value. Also, Change tags according to rules of type "if word-1 is a determiner and word is a verb then change the tag to noun" in a specific order. Training is done on tagged corpus by write a set of rule templates. Among the set of rules, find one with highest score then continue from 2 until lowest score threshold is passed. Keep the ordered set of rules, whereas rules make errors that are corrected by later rules as: Tagger labels every word with its most-likely tag. For example: race has the following probabilities in the Brown corpus: $\mathrm{P}(\mathrm{NN} \mid$ race $)=.98$ and $\mathrm{P}(\mathrm{VB} \mid \mathrm{race})=.02$ 
From the section 3, we got a preprocessed tagged file as output.txt, which can be used as an input file for the below logic for $\mathrm{xml}$ generation.

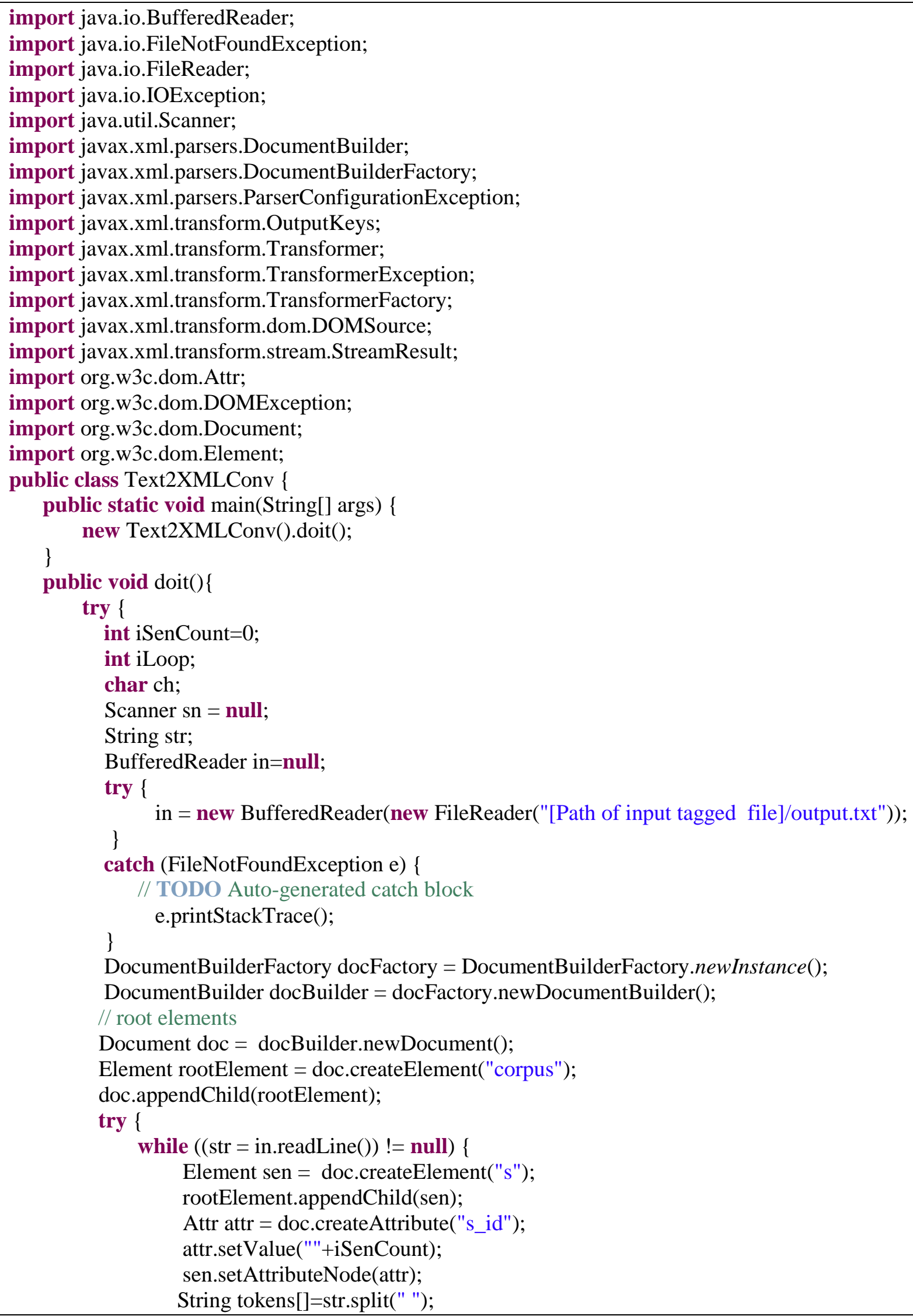




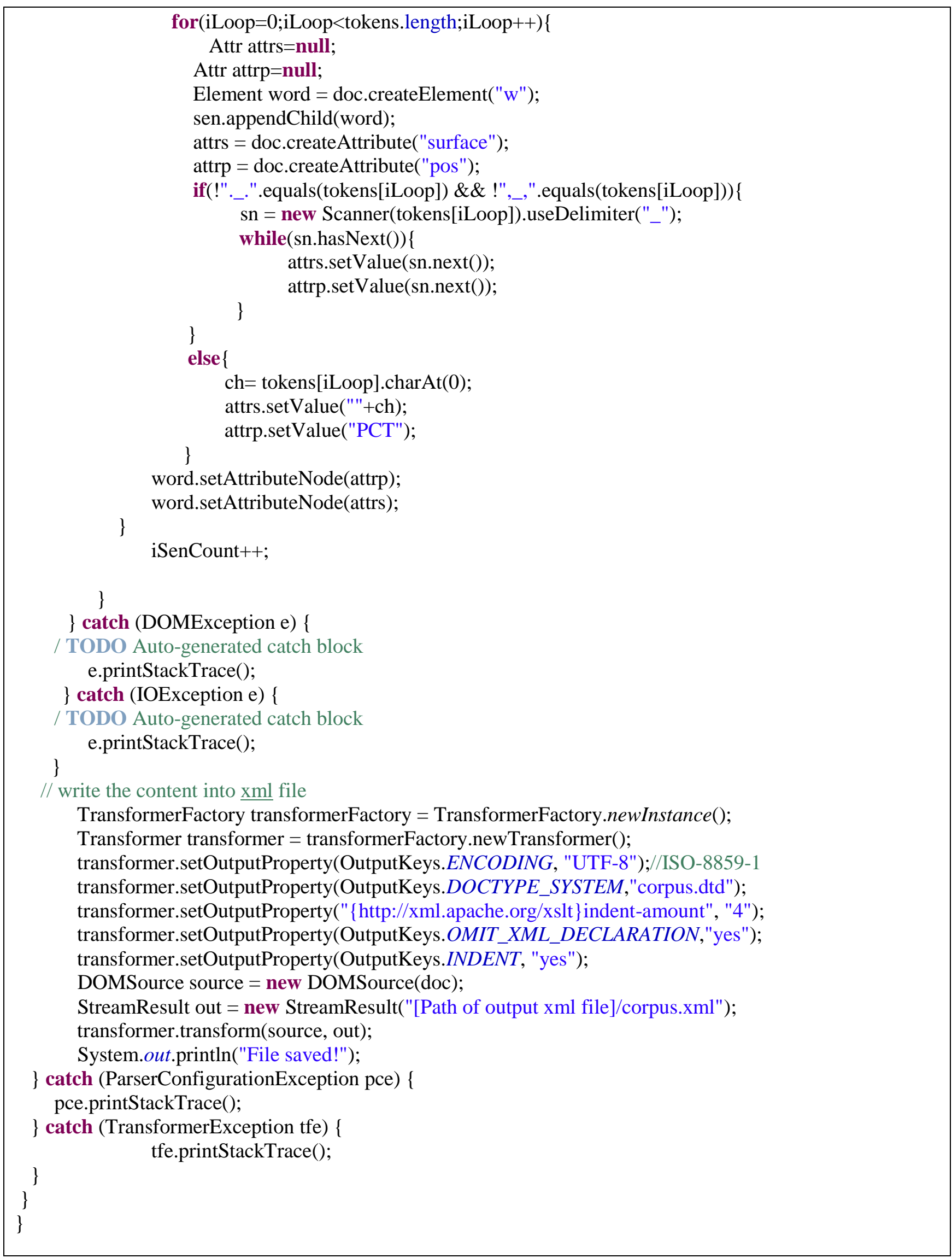


On successful execution of code in Figure 4.2, we would get corpus.xml as shown below in Figure 4.3.

$$
\begin{aligned}
& \text { DOCTYPE corpus (View Source for full doctype...) }> \\
& \text {-<corpus> } \\
& \text { - 〈s s_id="0"> } \\
& <w \text { pos="DT" surface="The" /> } \\
& <w \text { pos="NNS" surface="licenses" /> } \\
& <w \text { pos="IN" surface="for" / > } \\
& <w \text { pos="JJS" surface } \\
& \langle\text { w pos="NN" surface="software" / > } \\
& <\mathrm{w} \text { pos="VBP" surface="are" /> } \\
& <\mathrm{w} \text { pos="VBN" surface="designed" / > } \\
& <\mathrm{w} \text { pos="TO" surface="to" /> } \\
& <w \text { pos="VB" surface="take" /> } \\
& <w \text { pos="RP" surface="away" / > } \\
& <w \text { pos="PRP\$" surface="your" /> } \\
& <w \text { pos }=" \mathbf{N N "} \text { surface }=\text { "freedom" /> } \\
& <w \text { pos="TO" surface="to" /> } \\
& <w \text { pos="VB" surface="share" /> } \\
& <w \text { pos="CC" surface="and" / > } \\
& <w \text { pos="VB" surface="change" /> } \\
& <w \text { pos="PRP" surface="it" /> } \\
& <w \text { pos="PCT" surface="." /> } \\
& </ \text { s }>
\end{aligned}
$$

\section{CONCLUSION}

We are presenting an approach for generating corpus in $\mathrm{xml}$ format. It can be further used for Information Retrieval, TextTo-Speech conversion, Word Sense Disambiguation and also useful for preprocessing step of parsing by providing unique tag to each word which reduces the number of parses. Also, we are designing a toolkit for multiword extraction for a monolingual corpus. This paper is simply an initial step for the multiword extraction toolkit[6].

\section{REFERENCES}

[1] Andrew MacKinlay and Timothy Baldwin, "POS Tagging with a More Informative Tagset", at Proceedings of the Australasian Language Technology Workshop 2005, pages 40-48, Sydney, Australia, December 2005.
[2] Christopher D. Manning, Part-Of-Speech Tagging From 97\% To 100\%: Is It Time For Some Linguistics?, in CICLing2011.

[3] Su Cheng Haw, G. S. V. Radha Krishna Rao,,"A Comparative Study and Benchmarking on XML Parsers", Faculty of Information Technology, Multimedia University, 63100 Cyberjaya.

[4] Edwin Goei, Software Engineer, Sun Microsystems," Java and XML Parsing Using Standard APIs", September 11, 2000

[5] Nishchal Bhalla, Sahba Kazerooni,"Web Services Vulnerabilities", at Security Compass Inc 2007.

[6] C. Ramisch, A. Villavicencio, C. Boitet, Mwetoolkit: A Framework For Multiword Expression Identification", in: Proceedings of the Seventh International Conference on Language Resources and Evaluation (LREC 2010), Valetta, Malta, May 2010 